\title{
Alopecia Areata in Severe Atopic Dermatitis Treated With Dupilumab
}

Barroso-García B ${ }^{1}$, Rial $\mathrm{MJ}^{1,3}$, Molina $\mathrm{A}^{2}$, Sastre $\mathrm{J}^{1,3}$

${ }^{I}$ Allergy Department, Hospital Universitario Fundación Jiménez Diaz, Madrid, Spain

${ }^{2}$ Dermatology Department, Hospital Universitario Fundación Jiménez Díaz, Madrid, Spain

${ }^{3}$ CIBERES, Instituto de Salud Carlos III, Madrid, Spain

J Investig Allergol Clin Immunol 2018; Vol. 28(6): 420-421

doi: 10.18176/jiaci.0301

Key words: Alopecia areata. Atopic dermatitis. Dupilumab. Monoclonal antibody. Skin allergy.

Palabras clave: Alopecia areata. Dermatitis atópica. Dupilumab. Anticuerpo monoclonal. Alergia cutánea.

Dupilumab, a human monoclonal antibody against interleukin- 4 receptor $\alpha$, acts by inhibiting the signaling of interleukin-4 and interleukin-13, both of which are type 2 cytokines that may be important drivers of allergic or atopic diseases such as atopic dermatitis (AD) [1]. Dupilumab is the first biological therapy to be approved for treatment of moderate-to-severe AD in adults who are candidates for systemic therapy. Administration of dupilumab improves the signs and symptoms of $\mathrm{AD}$, including pruritus and anxiety and depression, and enhances patient quality of life [2,3]. A reassuring safety profile has been established, with conjunctivitis being the most significant safety signal [2-4]. We describe a patient with severe AD treated with dupilumab who developed alopecia areata within 6 weeks of his first exposure.

A 31-year-old white man with a 7-year history of severe $\mathrm{AD}$ and no other relevant past or family history presented with patches of hair loss on his anterior scalp. The patient had undergone previous treatment for AD consisting of phototherapy, topical and oral corticosteroids, cyclosporine, and azathioprine, although his response was poor. He was started on dupilumab at $600 \mathrm{mg}$ subcutaneously followed by $300 \mathrm{mg}$ subcutaneously every 2 weeks. After 6 weeks of treatment, his AD improved significantly; however, he noted hair loss in patches on his anterior scalp. Determination of the serum concentration of antithyroid antibodies, thyroglobulin, and thyroperoxidase yielded negative results. Thyroidstimulating hormone, $\mathrm{T} 3$, and free $\mathrm{T} 4$ determinations were also found to be normal. The results of a biopsy indicated alopecia areata with epidermal changes suggesting atopic dermatitis (areas of focal epidermal inflammation with exocytosis, spongiosis, and parakeratosis, which present as deep and patchy perifollicular lymphocytic infiltrate and frequent fibrotic tracts). The patient was diagnosed with alopecia areata, treatment with intralesional triamcinolone was initiated, and dupilumab was continued. Follow-up is required to assess the response of alopecia to corticosteroids. 


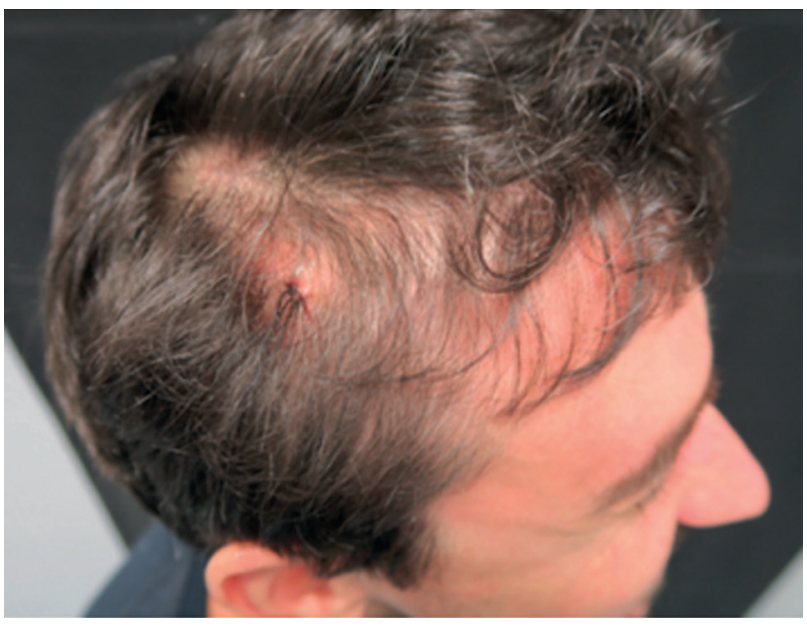

Figure. Development of alopecia areata during treatment with dupilumab. Clinical photograph of alopecia areata after 6 weeks of treatment and prior to treatment with intralesional triamcinolone acetonide.

To date, there has been only 1 reported case of alopecia areata following administration of dupilumab [5]. The patient was a man of a similar age to the patient we report, with a long history of severe $\mathrm{AD}$ and previous use of systemic drugs without response, who developed alopecia areata 5 weeks after initiation of dupilumab. However, as no biopsy was performed, the diagnosis of alopecia areata was purely clinical. Therefore, ours is the first report of biopsy-confirmed alopecia areata in a patient with severe AD treated with dupilumab.

The pathogenesis of alopecia areata is not completely understood, although several studies point to a heterogeneous process involving the $\mathrm{T}_{\mathrm{H}} 2$ response. In light of these findings, dupilumab is also being tested in a phase 2 clinical trial (NCT03359356) as a possible treatment for alopecia areata in patients with or without $\mathrm{AD}$ [6]. However, other immune system mediators, such as $\mathrm{T}_{\mathrm{H}} 1$, together with downregulation of $\mathrm{T}_{\mathrm{H}} 2$ pathways, may amplify the $\mathrm{T}_{\mathrm{H}} 1$ pathway and promote the development of alopecia areata after treatment with dupilumab [7]. Alopecia areata and AD have common immunological pathways and increased proinflammatory activation. Onset of both diseases concomitantly has been reported in a systematic review [8].

Clinical trials to date with dupilumab $[2,3,9]$ have not described alopecia areata as an adverse effect. These studies report only mild reactions, mainly conjunctivitis and injection site reactions. However, 2 clinical cases of alopecia areata have been reported during treatment with dupilumab in $\mathrm{AD}$ [5, present case]. Therefore, although the temporal relationship between administration of dupilumab and onset of alopecia areata does not ensure causality, the occurrence of both these events should raise suspicion. Therefore, it is important for clinicians to be aware that alopecia areata can occur in this context.

\section{Funding}

The authors declare that no funding was received for the present study.

\section{Conflicts of Interest}

J Sastre has served as a consultant to Thermo Fisher, MSD, Novartis, Genentech, Sanofi, Leti, Roche, FAES FARMA, Mundipharma, and GlaxoSmithKline. He has also received lecture fees from Novartis, GlaxoSmithKline, Stallergenes, LETI, and FAES FARMA and has received grant support for research from Thermo Fisher.

M Rial has served as a consultant to Allergy Therapeutics and Orion Pharma and has received lecture fees from Novartis, Astra Zeneca, Chiesi, and Merck.

B Barroso and A Molina declare that they have no conflicts of interest.

\section{References}

1. Werfel T, Allam JP, Biedermann T, Eyerich K, Gilles S, GuttmanYassky $\mathrm{E}$, et al. Cellular and molecular immunologic mechanisms in patients with atopic dermatitis. J Allergy Clin Immunol. 2016 Aug; 138(2):336-49.

2. Simpson EL, Bieber T, Guttman-Yassy E, Beck LA, Blauvelt A, Cork MJ, et al. Two Phase 3 Trials of Dupilumab versus Placebo in Atopic Dermatitis. N Engl J Med. 2016 Dec 15;375(24):2335-48.

3. Blauvelt $A$, de Bruin-Weller $M$, Gooderham $M$, Cather JC, Weisman J, Pariser $D$, et al. Long-term management of moderate-to-severe atopic dermatitis with dupilumab and concomitant topical corticosteroids (LIBERTY AD CHRONOS): a 1-year, randomised, double-blinded, placebo-controlled, phase 3 trial. Lancet. 2017 Jun 10;389(10086):2287-03.

4. Gooderham MJ, Hong HC, Eshtiaghi P, Papp KA. Dupilumab: A review of its use in the treatment of atopic dermatitis. J Am Acad Dermatol. 2018 Mar;78(351):S28-36.

5. Mitchell K, Levitt J. Alopecia areata after dupilumab for atopic dermatitis. JAAD Case Rep. 2018 Jan 16:4(2):143-4.

6. https://clinicaltrials.gov/ct2/show/NCT03359356 llast accessed: April 26, 2018).

7. Revert-Yuval Y, Guttman-Yassky E. The changing landscape of alopecia areata: the therapeutic paradigm. Adv Ther. 2017;34:1594-9.

8. Mohan GC, Silverberg Jl. Association of vitiligo and alopecia areata with atopic dermatitis: a systematic review and metaanalysis. JAMA Dermatol. 2014;151:522-8.

9. Sastre J, Dávila I. Dupilumab: A new paradigm for the treatment of allergic diseases. J Investig Allergol Clin Immunol. 2018 Jun;28(3):139-50.

Manuscript received May 23, 2018; accepted for publication August 1, 2018.

Manuel Jorge Rial Prado Allergy Department Hospital Universitario Fundación Jiménez Díaz Madrid, Spain Email: manuel.rial@quironsalud.es 